\title{
Manipulating free-space optical beams with a silicon photonic mesh
}

\author{
Maziyar Milanizadeh ${ }^{1}$, Piero Borga ${ }^{1}$, Francesco Morichetti ${ }^{1}$, D.A.B. Miller ${ }^{2}$, Andrea Melloni ${ }^{1}$ \\ ${ }^{1}$ Dipartimento di Elettronica, Informazione e Bioingegneria - Politecnico di Milano, Milano, 20133 Italy. \\ ${ }^{2}$ Ginzton Laboratory, Stanford University, Spilker Building, Stanford, CA 94305, USA \\ mail: andrea.melloni@polimi.it
}

\begin{abstract}
Photonic integrated meshes made of tuneable interferometers enable implementation of functions programmable on demand and are being envisioned as the optical counterpart of electronic field programmable gate arrays. Several examples of photonic processors capable of performing arbitrary linear operations have been recently proposed, and are expected to find applications in different areas, from the on-chip processing of telecom signals to microwave photonics, and from quantum optics to neural networks. In this work, we use a reconfigurable mesh of silicon photonic Mach-Zehnder Interferometers (MZIs) to manipulate free-space optical beams. We demonstrate beam steering, beam coupling from a free-space optical source to a single mode waveguide and automatic identification of the direction of arrival of a beam from a free-space source.
\end{abstract}

Keywords: silicon photonics, reconfigurable photonic integrated circuits, free-space optics

(a)

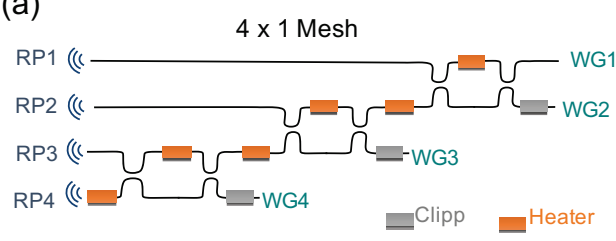

(b) $\quad 4 \times 1$ Mesh

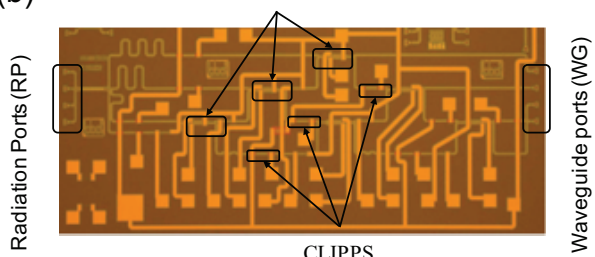

Figure 1: (a) Schematic representation of a 4 x 1 triangular mesh and (b) its implementation on a conventional silicon photonic platform. The overall footprint of the circuit is $3.7 \mathrm{~mm}$ by $1.5 \mathrm{~mm}$.

\section{INTRODUCTION}

Silicon photonics (SiP) is a promising platform for the realization of large scale integration of photonic processors, not only because of the high degree of device miniaturization, but also because of the possibility of integrating fast and power-efficient tuning elements and on-chip monitor photodetectors, which are necessary for robust reconfiguration and control of the photonic architecture. In this work, we demonstrate that a SiP mesh of Mach-Zehnder Interferometers (MZIs) can be effectively used to manipulate free-space optical beams. A number of optical functions are implemented, including beam steering and identification and coupling of a free-space beam coming from an arbitrary direction.

\section{SILICON PHOTONIC MESH}

The photonic integrated circuit (PIC) consists of a triangular mesh of Mach-Zehnder Interferometers (MZIs) arranged as in the scheme of Fig. 1a, which was fabricated on a standard $220 \mathrm{~nm}$ SiP platform by using $500 \mathrm{~nm}$ wide channel waveguides (see Fig. 2a). The four ports labelled as RPi consist of an array of radiating elements (grating couplers) with a mutual spacing of $127 \mu \mathrm{m}$ to either couple a free-space optical beam into the photonic chip or to radiate a guided-wave signal out to free space. All the balanced MZIs integrate $3 \mathrm{~dB}$ directional couplers with a gap of $300 \mathrm{~nm}$ and a length of $40 \mu \mathrm{m}$. Two phase shifters are integrated in each MZI by using TiN metal strips $(1 \mu \mathrm{m} \times 100 \mu \mathrm{m})$ to implement amplitude- and phase-tuneable couplers. At the output port of each MZI, onchip photodetectors (here realized through CLIPP transparent detectors using the same TiN used for thermal tuners) are used to locally monitor the switching state of each MZI and implement automatic tuning and

(a)

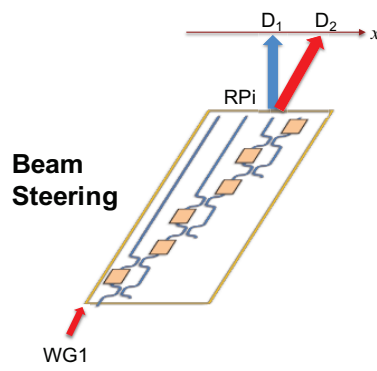

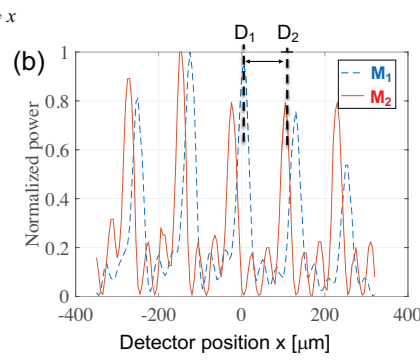

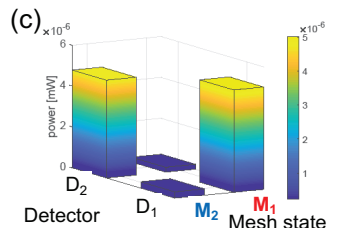

Figure 2: (a) Schematic representation of the beam steering experiment. (b) Free-space radiation pattern measured at the distance of 11 mm from the grating array RPi when the light is coupled to input port WG1 and the mesh is tuned in two different states $\mathrm{M}_{1}$ and $\mathrm{M}_{2}$. (c) Optical power measured by a detector (collimator) placed at positions $D_{1}$ and $D_{2}$ when the mesh is configured in states $M_{1}$ and $M_{2}$ 
stabilization procedures. More details on the circuit design, fabrication technology and the electronic circuit for the automatic control of the mesh can be found in [1]. All the experiments reported in this work were carried out at a wavelength of $1530 \mathrm{~nm}$.

\section{STEERING, COUPLING AND IDENTIFICATION OF A FREE-SPACE OPTICAL BEAM}

The SiP mesh was employed to manipulate free-space optical beams, and, in detail, the following functionalities were demonstrated:

(a) Beam steering. As a first application we used the mesh to demonstrate the steering of a free-space optical beam. To this end, we injected into the mesh an optical signal from port WG1 (see Fig. 2a) and we measured the radiation pattern generated by the grating array $(\mathrm{RPi})$ for different tuning states of the mesh. The radiation pattern was measured by sampling the optical field along the transverse $\mathrm{x}$-axis with a probe fiber with a resolution $d x=5$ $\mu \mathrm{m}$. Figure 2(b) shows the radiated field at a distance of $(11 \mathrm{~mm})$ from the output ports when the mesh is tuned in order to maximize the power received by a first detector $\mathrm{D}_{1}$ (placed at $x=0$, mesh state $\mathrm{M}_{1}$ ) and by a second detector $\mathrm{D}_{2}\left(x=100 \mu \mathrm{m}\right.$, mesh state $\left.\mathrm{M}_{2}\right)$. The spacing between the maxima of the field pattern (about $150 \mu \mathrm{m}$, corresponding to an angular spacing of about $\left.1^{\circ}\right)$ is related to the large physical distance $(127 \mu \mathrm{m})$ between radiating elements. Figure 2(c) shows the optical power collected by a detector ( $1 \mathrm{~mm}$ fiber collimator spaced 51.5 $\mathrm{cm}$ away from the chip) placed at the same angular direction of D1 and D2, when the mesh is tuned in states $\mathrm{M}_{1}$ and $\mathrm{M}_{2}$. Though the steering angle is rather limited, this demonstrates high resolution in the control of the radiation direction.

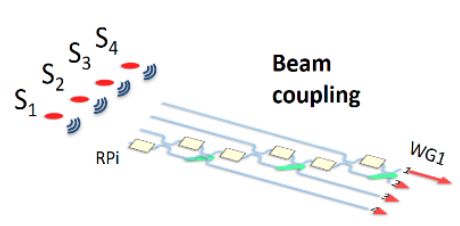

(a)

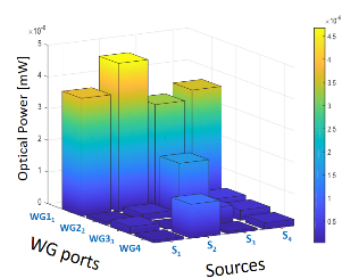

(b)

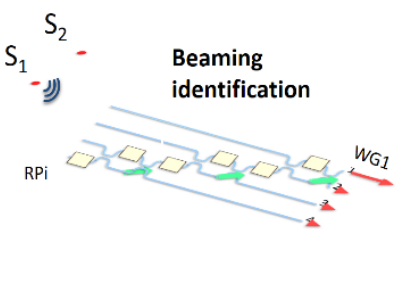

(c)

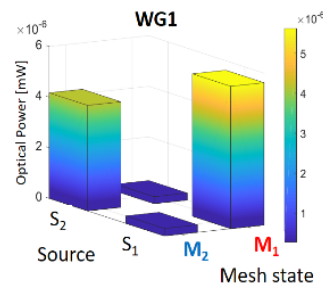

(d)

Figure 3: (a) Schematic representation of the beam coupling experiment. (b) Optical power coupled at the waveguide ports ( $\mathrm{WG}_{1}$-WG $\mathrm{W}_{4}$ ) when the mesh is configured to couple the light from an arbitrary free-space source $\left(\mathrm{S}_{1}-\mathrm{S}_{4}\right)$ to port WG1. (c) Schematic representation of the beam identification experiment. (d) Optical power coupled at port WG1 when the mesh automatically self-configures to maximize the power coming from an unknown source $\left(\mathrm{S}_{1}\right.$ or $\left.\mathrm{S}_{2}\right)$.

(b) Beam coupling and source identification. The high angular resolution of the mesh can be exploited to couple a free-space beam, coming from an arbitrary direction of arrival, into a single-mode optical waveguide while minimizing the coupling from other directions. To demonstrate this concept, four free-space optical beams $\left(\mathrm{S}_{1}-\mathrm{S}_{4}\right)$ were simultaneously shone on the grating array RPi by using an array of 4 fibers spaced by $d=127 \mu \mathrm{m}$ at a distance of $5.5 \mathrm{~mm}$ from the mesh (see Fig. 3a). When a beam from a given fiber impinges on the grating array, the mesh automatically self-configures by following a progressive tuning scheme [1] in order to maximize the transmission to port WG1. Figure 3(b) shows the power coupled to the waveguide ports WGi when the mesh is configured to couple the light from $\mathrm{S}_{1}-\mathrm{S}_{4}$ to WG1. This feature implies that the mesh can automatically identify the direction from which a beam is arriving. In Fig. 3(c)-(d) a beam is coming from a source whose position is not known (it can either be $\mathrm{S}_{1}$ or $\mathrm{S}_{2}$ ). The mesh automatically self-configures to maximize the coupling to WG1 and once convergence is achieved, the phase shifts (voltages) applied to the thermal tuners of the MZIs of the mesh identify the position of the source.

\section{CONCLUSION}

We demonstrate manipulation of free-space optical beams by using a 4x1 self-configuring SiP mesh. The performance of the mesh in steering, coupling and identifying free-space beams can be improved by optimizing the design of the radiating elements, whose number can be scaled up without impairing the progressive selfconfiguration procedure employed for the tuning of the mesh. Applications are envisioned to more advanced freespace optical processing, including phase front reconstruction, beaming through scattering media and chip-to-chip free-space communications.

This work was partially supported by the European Commission through the H2020 project Super Pixel (grant 829116) and by Fondazione Cariplo through the project ACTIO (grant 2016-0881).

\section{REFERENCES}

[1] Annoni, A. et al. Unscrambling light—automatically undoing strong mixing between modes. Light: Science \& Applications 6, e17110 (2017). 